\title{
Scattering amplitudes, black holes and leading singularities in cubic theories of gravity
}

\author{
William T. Emond ${ }^{a}$ and Nathan Moynihan ${ }^{b}$ \\ ${ }^{a}$ School of Physics and Astronomy, University of Nottingham, University Park, \\ Nottingham NG7 2RD, United Kingdom \\ ${ }^{b}$ The Laboratory for Quantum Gravity $\&$ Strings, \\ Department of Mathematics \& Applied Mathematics, University Of Cape Town, \\ Rondebosch, Cape Town 7r00, South Africa \\ E-mail: william.emond@nottingham.ac.uk, nathantmoynihan@gmail.com
}

ABSTRACT: We compute the semi-classical potential arising from a generic theory of cubic gravity, a higher derivative theory of spin-2 particles, in the framework of modern amplitude techniques. We show that there are several interesting aspects of the potential, including some non-dispersive terms that lead to black hole solutions (including quantum corrections) that agree with those derived in Einsteinian cubic gravity (ECG). We show that these non-dispersive terms could be obtained from theories that include the GaussBonnet cubic invariant $G_{3}$. In addition, we derive the one-loop scattering amplitudes using both unitarity cuts and via the leading singularity, showing that the classical effects of higher derivative gravity can be easily obtained directly from the leading singularity with far less computational cost.

Keywords: Scattering Amplitudes, Classical Theories of Gravity, Models of Quantum Gravity

ARXIV EPRINT: 1905.08213 


\section{Contents}

1 Introduction 1

2 Cubic theories of gravity 2

3 Scattering amplitudes and the effective potential 3

4 One loop amplitude $\quad 4$

4.1 Unitarity cuts 4

$\begin{array}{lll}4.2 & \text { Leading singularity } & 7\end{array}$

5 Potential and black hole solutions 13

$\begin{array}{lll}6 & \text { Discussion } & 15\end{array}$

A Tree-level 4-point amplitude $\quad 16$

$\begin{array}{ll}\text { B Fourier transforms } & 17\end{array}$

\section{Introduction}

The modern S-matrix program has been wildly successful when applied to gravitation [14], including higher derivative theories [5-9]. In the age of LIGO, there has been much attention given to the simplicity of amplitude techniques in computing post-Newtonian and post-Minkowskian corrections to General Relativity (GR) [10, 11], and in fact it has been shown that the classical contribution of loop amplitudes correspond to terms in a postMinkowskian expansion [12, 13]. As an interesting modification of GR, one can consider theories of gravity which involve terms cubic in either the Riemann or Ricci tensors which, among other things, contain non-trivial black hole solutions in four dimensions [14]. Such higher-derivative contributions to the gravitational action are often encountered within string theory [15], and can be formulated in such a way as they only possess spin-2 degrees of freedom on-shell $[16,17]$. In this paper, we will explore both classical and quantum aspects of this theory by computing its scattering amplitudes using modern techniques. From there, we will use these amplitudes to derive the semi-classical potential associated with cubic theories of gravity, where the purely classical graviton mediated interaction between two scalars is affected by cubic terms only at one-loop order and above.

In section 2 we review cubic theories of gravity - including Einsteinian cubic gravity in order to setup the problem we will consider. In section 3, we develop the tools required to obtain the semi-classical potential and black hole solutions directly from scattering 
amplitudes before moving on to section 4.1, where we compute the massive scalar oneloop amplitude using unitarity cuts. This requires us to compute the coefficients of the standard integrals that usually arise in a Passarino-Veltman loop decomposition, from which we derive the quantum- corrected classical potential and the classical and quantum corrections to the Schwarzschild black hole solution arising from the addition of a cubic term in the gravitational action. Computing amplitudes via unitarity cuts is computationally expensive, and so to contrast this, in section 4.2 we derive the classical contribution of the amplitude directly from the Leading singularity [18], where loop integration is reduced to the far simpler problem of computing residues.

\section{Cubic theories of gravity}

Higher derivative operators in gravity are important for a variety of reasons, including the modification of gravity at short distances/large energies and the possibility of renormalizability. One particularly interesting theory of gravity in this class is Einsteinian cubic gravity (ECG) [14, 16], which enjoys the same linearised spectrum as General Relativity, in that it propagates only two degrees of freedom on-shell.

We will consider a generic six-derivative theory in four dimensions described by the action

$$
S=\int \mathrm{d}^{4} x \sqrt{-g}\left(\frac{2}{\kappa^{2}} R+\lambda \mathcal{P}\right)
$$

where the coupling has mass dimension $[\lambda]=-2$ and

$$
\mathcal{P}=\beta_{1} R^{\mu}{ }_{\alpha \nu \beta} R^{\alpha \lambda \beta \sigma} R_{\lambda \mu \sigma}{ }^{\nu}+\beta_{2} R_{\mu \nu}{ }^{\alpha \beta} R_{\alpha \beta}{ }^{\lambda \sigma} R_{\lambda \sigma}{ }^{\mu \nu}+\beta_{3} R_{\mu \nu \alpha \beta} R^{\mu \alpha} R^{\nu \beta}+\beta_{4} R_{\mu}{ }^{\nu} R_{\nu}{ }^{\alpha} R_{\alpha}{ }^{\mu} .
$$

We leave these coefficients generic, in order to keep track of how each of these terms contributes to the physical effects, however, when required to specialise to ECG, we will consider the specific set of coefficients

$$
\beta_{1}=12, \beta_{2}=1, \beta_{3}=-12, \beta_{4}=8 .
$$

Nonetheless, as is well known, only the first two terms typically contribute to the S-matrix at cubic order, and furthermore, a specific choice of coefficients, $\beta_{1}=-2 \beta_{2}$, gives the well known cubic Gauss-Bonnet invariant

$$
G_{3}=R_{\mu \nu}{ }^{\alpha \beta} R_{\alpha \beta}{ }^{\lambda \sigma} R_{\lambda \sigma}{ }^{\mu \nu}-2 R^{\mu \nu}{ }_{\alpha \nu} R^{\alpha \lambda \beta \sigma} R_{\lambda \mu \sigma}{ }^{\nu} .
$$

While this term does not produce pure graviton dynamics on its own, when coupled to Einstein gravity or generic matter, it can produce non-trivial scattering effects $[19,20]$. As expected from a cubic theory of gravity, with the predictable coefficients expected given the argument above, the on-shell three-point all minus graviton amplitude at order $\lambda$ is given by

$$
M^{---}=\frac{3}{8} \kappa^{3} \lambda\left(\beta_{1}+2 \beta_{2}\right)\langle 12\rangle^{2}\langle 23\rangle^{2}\langle 31\rangle^{2},
$$

where we have derived this using eq. (A.2) contracted with a graviton polarization tensor. At tree level, when compared with the contributions from General relativity (GR), we 
find that although the three-point vertex itself is modified by the $\mathcal{O}\left(R^{3}\right)$ terms, we do not modify the scalar-scalar-graviton vertex. This means that at first-order in $G$ the Newtonian potential must be the same in both GR and ECG, and thus we would expect any static, spherically symmetric black hole solution to be a higher-order perturbation of the Schwarzschild solution. To find such a higher order contribution then, we must compute the classical contributions that arise from a one-loop amplitude, and from this, derive the classical potential.

\section{Scattering amplitudes and the effective potential}

A particularly sensible definition of the potential energy is to define it in terms of gaugeinvariant on-shell scattering amplitudes in the non-relativistic limit. To this end, we will consider $2 \longrightarrow 2$ scattering of two massive scalars mediated by gravity. This ensures that the definition of the potential is itself gauge-invariant [21] and that, in the non-relativistic limit $\left(t=-\mathbf{q}^{2}\right)$, can be given by the inverse Born approximation [22, 23]

$$
V(\mathbf{r}, \mathbf{p})=-\frac{1}{4 E_{A} E_{B}} \int \frac{\mathrm{d}^{3} \mathbf{q}}{(2 \pi)^{3}} e^{i \mathbf{q} \cdot \mathbf{r}} \mathcal{M}(\mathbf{q}, \mathbf{p}),
$$

where $\mathbf{q}$ is the exchanged three-momentum and $E_{A}\left(E_{B}\right)$ is the energy associated with particle $A(B)$.

Ultimately, we would like to derive the metric associated to a black hole in an asymptotically flat spacetime $[24,25]$, meaning we need to relate the potential energy to the metric. We will therefore consider the gravitational field to be sourced by two point-masses in the stationary limit. In this limit, the usual relativistic action for a point particle is only dependent on $g_{00}$ and we therefore consider the following path integral [26]

$$
Z=\int \mathcal{D} h_{\mu \nu} \exp \left[-i\left(S_{E H}+m_{A} \int_{-T / 2}^{T / 2} d \sigma \sqrt{g_{00}(\sigma)}+m_{B} \int_{-T / 2}^{T / 2} d \sigma^{\prime} \sqrt{g_{00}\left(\sigma^{\prime}\right)}\right)\right] .
$$

This describes the two sources, with masses $m_{A}$ and $m_{B}$, interacting at rest on a flat background. They begin at some fixed distance, with their interaction adiabatically turned on at a (large) time $-T / 2$, and turned off at $T / 2$. We can then consider the generating functional given by

$$
\begin{aligned}
\mathcal{F} & =\frac{\int \mathcal{D} h_{\mu \nu} \exp \left[-i\left(S_{E H}+m_{A} \int_{-T / 2}^{T / 2} d \sigma \sqrt{g_{00}(\sigma)}+m_{B} \int_{-T / 2}^{T / 2} d \sigma^{\prime} \sqrt{g_{00}\left(\sigma^{\prime}\right)}\right)\right]}{\int \mathcal{D} h_{\mu \nu} \exp \left[-i S_{E H}\right]} \\
& =\left\langle\exp \left[-i\left(m_{A} \int_{-T / 2}^{T / 2} d \sigma \sqrt{g_{00}(\sigma)}+m_{B} \int_{-T / 2}^{T / 2} d \sigma^{\prime} \sqrt{g_{00}\left(\sigma^{\prime}\right)}\right)\right]\right\rangle .
\end{aligned}
$$

In the $T \longrightarrow \infty$ limit, this is well approximated by the ground state energy [26], meaning we can say that $\lim _{T \rightarrow \infty} \mathcal{F} \sim e^{-i V(R) T}$, and we can define the potential energy via

$$
V(r)=\lim _{T \longrightarrow \infty} \frac{i}{T} \log (\mathcal{F}) \simeq \lim _{T \longrightarrow \infty} \frac{1}{T}\left(m_{A} \int_{-T / 2}^{T / 2} d \sigma \sqrt{g_{00}(\sigma)}+m_{B} \int_{-T / 2}^{T / 2} d \sigma^{\prime} \sqrt{g_{00}\left(\sigma^{\prime}\right)}\right) .
$$


In the static, spherically symmetric limit in which we are interested, the potential $\Phi$ is related to the metric via

$$
g_{00}=1-2 \Phi .
$$

If we consider the probe limit where $m_{B} \ll m_{A}$, then we can discard the gravitational field produced by $m_{B}$ and easily perform the integral in eq. (3.4) for the static case (where we take $|\Phi| \ll 1)$, to find

$$
V(r)=m_{B} \sqrt{1-2 \Phi} \simeq m_{B}(1-\Phi) .
$$

Deriving the potential energy $V(r)$ from the amplitudes, then, allows us to compute the potential directly. One way to do this is to expand $\Phi\left(r, m_{A}\right)$ in terms of $G$ as

$$
\Phi\left(r, m_{A}\right)=\sum_{n=1}^{\infty} C_{n}\left(m_{A}, r\right) G^{n}
$$

where $C_{n}\left(r, m_{A}\right)$ will be a combination of $m_{A}$ and $r$ with mass dimension 2 . We can then directly compare this order by order with the potential energy in the correct limit

$$
\Phi\left(r, m_{A}\right)=\sum_{n=1}^{\infty} C_{n}\left(r, m_{A}\right) G^{n}=-\lim _{m_{B} \longrightarrow 0} \frac{1}{m_{B}}\left(\frac{1}{m_{A} m_{B}} \int \frac{\mathrm{d}^{3} \mathbf{q}}{(2 \pi)^{3}} e^{i \mathbf{q} \cdot \mathbf{r}} \mathcal{M}(\mathbf{q})\right)
$$

With this solution in hand, the line element is given by

$$
d s^{2}=-(1-2 \Phi) d t^{2}+(1+2 \Phi) d r^{2}+r^{2} d \Omega
$$

\section{One loop amplitude}

\subsection{Unitarity cuts}

We will first compute the relevant one-loop amplitude using standard on-shell unitarity cuts. As is well known, corrections to the potential arise from the purely non-analytic pieces of loop amplitudes, corresponding to long-range effects of massless particle interactions [27]. This means that we only need to consider cuts in the $t$-channel, and we need not consider all possible cuts. Indeed, we are free to ignore those that will give purely analytic contributions to the amplitudes. The non-analytic pieces of loop amplitudes are also independent of regularization scheme, and as such we can happily work in $D=4$ throughout the calculation [28]. Since only the graviton three-point vertex is modified in cubic theories, the one-loop box diagram must be the same as it is in GR, and thus we will focus first on the triangle diagram, noting that any contributions ought to come from diagrams containing massive propagators, which facilitate the delicate $\hbar$ cancellations that give rise to purely classical pieces $[27,29]$. We will consider the diagram given in figure 2 . To compute the double cut, we need to evaluate

$$
M_{4}^{(1)}=-\left.i \sum_{h_{1}, h_{2}} \int \frac{\mathrm{d}^{4} \ell_{1}}{(2 \pi)^{4}} \frac{M_{L}\left[P_{1}, P_{2}, \ell_{1}^{h_{1}}, \ell_{2}^{h_{2}}\right] M_{R}\left[-\ell_{1}^{-h_{1}},-\ell_{2}^{-h_{2}}, P_{3}, P_{4}\right]}{\ell_{1}^{2} \ell_{2}^{2}}\right|_{\ell_{1}^{2}=\ell_{2}^{2}=0},
$$




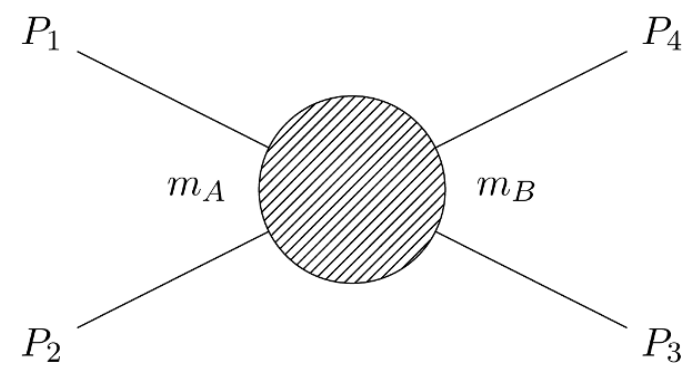

Figure 1. The kinematic setup, where particles 1 and 4 are incoming and 2 and 3 outgoing. In the center of mass frame, we consider the exchanged momentum $q=P_{1}+P_{2}=(0, \mathbf{q})$ (in the all outgoing convention) and $P_{1}=\left(E_{A}, \mathbf{q} / 2\right), P_{2}=-\left(E_{A},-\mathbf{q} / 2\right)$ with $E_{A}=\sqrt{m_{A}^{2}+\mathbf{p}^{2}+\frac{\mathbf{q}^{2}}{4}} \cdot P_{3}$ and $P_{4}$ are defined similarly with $A \leftrightarrow B$.

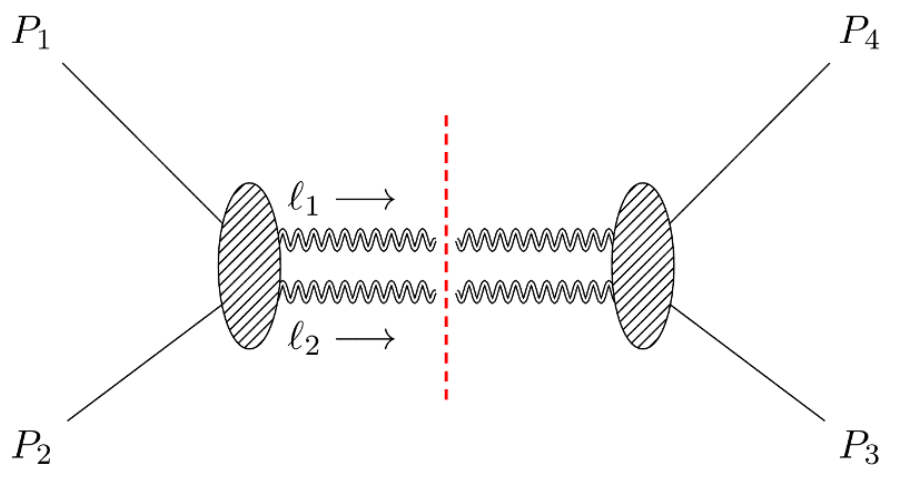

Figure 2. Double Cut Diagram.

where $\ell_{2}=\ell_{1}-P_{1}-P_{2}$ and $P_{1}^{2}=P_{2}^{2}=m_{A}^{2}, P_{3}^{2}=P_{4}^{2}=m_{B}^{2}$. The cut conditions are therefore given by

$$
\begin{aligned}
\ell_{1}^{2} & =\left(\ell_{1}-q\right)^{2}=0 \\
& \Rightarrow \quad 2 \ell_{1} \cdot q=q^{2}
\end{aligned}
$$

where $q=P_{1}+P_{2}$. Note that eq. (4.2) implies that $P_{2} \cdot \ell_{1}=m_{A}^{2}+P_{1} \cdot P_{2}-P_{1} \cdot \ell_{1}=\frac{t}{2}-P_{1} \cdot \ell_{1}$. Moreover, we define the Mandelstam variable $t=q^{2}=\left(P_{1}+P_{2}\right)^{2}=\left(\ell_{1}+\ell_{2}\right)^{2}=2 \ell_{1} \cdot \ell_{2}$, using the kinematic conventions set out in figure 1 . The tree-level amplitudes are given in figure 3. The tree level diagrams on both sides of the cut are the classical gravitational Compton diagrams, given by

At order $\lambda^{1}$ in the three-graviton coupling (i.e. ignoring the $\lambda^{0}$ GR contribution and $\lambda^{2}$ pure cubic contributions to eq. (4.3)), and choosing to focus on the $h_{1}=h_{2}=-$ case, the corresponding amplitudes are given by

$$
\begin{aligned}
& M_{L}\left[P_{1}, P_{2}, \ell_{1}^{-}, \ell_{2}^{-}\right]^{(G R)}= \frac{\kappa^{2}}{16} \frac{m_{A}^{4}\left\langle\ell_{1} \ell_{2}\right\rangle^{4}}{\left(P_{1}+P_{2}\right)^{2}\left(P_{1} \cdot \ell_{1}\right)\left(P_{2} \cdot \ell_{1}\right)}, \\
& M_{R}\left[-\ell_{1}^{+},-\ell_{2}^{+}, P_{3}, P_{4}\right]^{\left(R^{3}\right)}=\frac{3}{16} \frac{\kappa^{4} \lambda\left[\ell_{1} \ell_{2}\right]^{4}}{\left(P_{3}+P_{4}\right)^{2}}\left(\beta_{1}\left(\left(\ell_{1} \cdot P_{3}-\ell_{1} \cdot P_{4}\right)^{2}-m_{B}^{2} \ell_{1} \cdot \ell_{2}\right)\right. \\
&\left.-8 \beta_{2}\left(\ell_{1} \cdot P_{3}\right)\left(\ell_{1} \cdot P_{4}\right)\right) .
\end{aligned}
$$



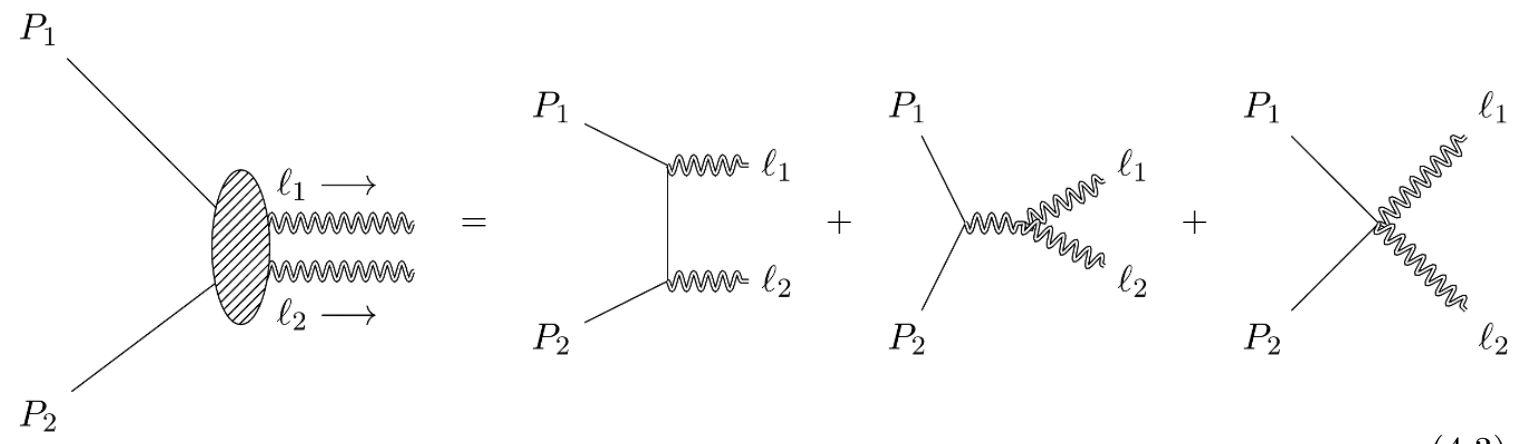

Figure 3. Tree Diagrams.

The loop amplitude is therefore given by ${ }^{1}$

$$
M_{4}^{(1)}=\frac{3 \kappa^{6} \lambda m_{A}^{4} t^{2}}{32} \int \frac{\mathrm{d}^{4} \ell}{(2 \pi)^{4}} \frac{\beta_{1}\left(\left(\ell \cdot P_{3}-\ell \cdot P_{4}\right)^{2}-\frac{1}{2} m_{B}^{2}\left(P_{3}+P_{4}\right)^{2}\right)-8 \beta_{2}\left(\ell \cdot P_{3}\right)\left(\ell \cdot P_{4}\right)}{\ell^{2}\left(\ell-P_{1}-P_{2}\right)^{2}\left[\left(\ell-P_{1}\right)^{2}-m_{A}^{2}\right]\left[\left(\ell-P_{2}\right)^{2}-m_{A}^{2}\right]} .
$$

Performing a standard Passarino-Veltman decomposition, we can express the loop amplitude as a sum of boxes, triangles and bubbles. We find that there are no box contributions, but that there are triangles and bubbles. Evaluating these with the help of Package-X [30], we find

$$
\begin{aligned}
M_{4}^{(1)}=-\frac{3 \kappa^{6} \lambda m_{A}^{4} t^{2}}{32\left(t-4 m_{A}^{2}\right)^{2}} & {\left[\left(\beta_{1}+2 \beta_{2}\right) b_{2}(t) B_{0}(t)\right.} \\
& \left.+\left[\left(\beta_{1}+2 \beta_{2}\right) c_{3}^{2}(t)+\beta_{1} c_{3}^{1}(t)\right] C_{0}\left(P_{1}^{2}, P_{2}^{2}, t ; 0, m_{A}, 0\right)\right]
\end{aligned}
$$

where $B_{0}$ and $C_{0}$ are the bubble and triangle scalar Passarino-Veltman functions and

$$
\begin{aligned}
& b_{2}(t)= 6 m_{A}^{4}+4 m_{A}^{2}\left(m_{B}^{2}-3 s\right)+6\left(m_{B}^{2}-s\right)^{2}-2\left(2\left(m_{A}^{2}+m_{B}^{2}\right)-3 s\right) t+t^{2}, \\
& c_{3}^{1}(t)= \frac{1}{2}\left(t-4 m_{A}^{2}\right)^{2}\left(t-2 m_{B}^{2}\right), \\
& c_{3}^{2}(t)=2\left[2 m_{A}^{2}\left(s-\left(m_{A}-m_{B}\right)^{2}\right)\left(s-\left(m_{A}+m_{B}\right)^{2}\right)+\left(-3 m_{A}^{4}+2 m_{A}^{2} m_{B}^{2}+\left(m_{B}^{2}-s\right)^{2}\right) t\right. \\
&\left.\quad+\left(m_{A}^{2}-m_{B}^{2}+s\right) t^{2}\right] .
\end{aligned}
$$

Expanding these terms for small $t$ (and keeping only the terms that give rise to leading

\footnotetext{
${ }^{1}$ For the purposes of isolating the contribution of the $G_{3}$ term, one can express the four point as

$$
M_{R}\left[-\ell_{1}^{+},-\ell_{2}^{+}, P_{3}, P_{4}\right]^{\left(R^{3}\right)}=\frac{3}{64} \kappa^{4} \lambda\left[\ell_{1} \ell_{2}\right]^{4} \frac{\beta_{1} t\left(t-2 m_{A}^{2}\right)-16\left(\beta_{1}+2 \beta_{2}\right)\left(\ell_{1} \cdot P_{3}\right)\left(\ell_{1} \cdot P_{3}\right)}{\left(P_{3}+P_{4}\right)^{2}} .
$$
}




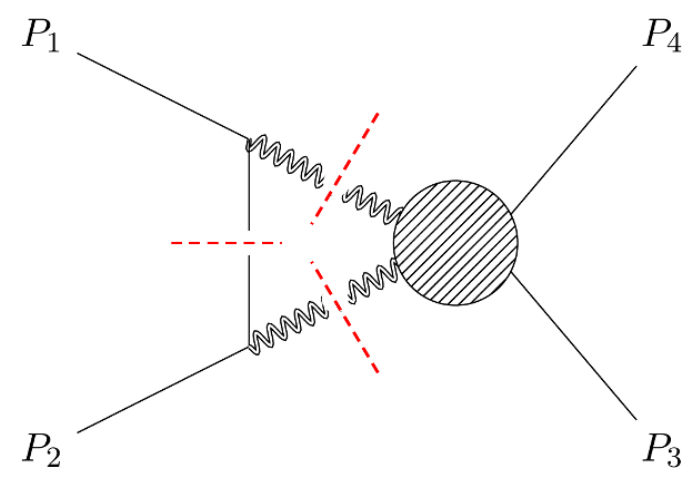

Figure 4. LS Triangle Diagram.

order classical and quantum contributions to the potential) we find

$$
\begin{aligned}
\frac{t^{2} b_{2}(t)}{\left(t-4 m_{A}^{2}\right)^{2}} & =\left(3 m_{A}^{4}+2 m_{A}^{2}\left(m_{B}^{2}-3 s\right)+3\left(m_{B}^{2}-s\right)^{2}\right) \frac{t^{2}}{8 m_{A}^{4}}+\mathcal{O}\left(t^{3}\right), \\
\frac{t^{2} c_{3}^{1}(t)}{\left(t-4 m_{A}^{2}\right)^{2}} & =-m_{B}^{2} t^{2}+\frac{1}{2} t^{3} \\
\frac{t^{2} c_{3}^{2}(t)}{\left(t-4 m_{A}^{2}\right)^{2}} & =\left(s-\left(m_{A}-m_{B}\right)^{2}\right)\left(s-\left(m_{A}+m_{B}\right)^{2}\right) \frac{t^{2}}{m_{A}^{2}}+\mathcal{O}\left(t^{3}\right) .
\end{aligned}
$$

\subsection{Leading singularity}

Computing scattering amplitudes via the unitarity cuts method is an often cumbersome (or impossible) affair, requiring us to solve complicated divergent loop integrals using some regularisation scheme or other and perhaps a clever technique for integrand reduction. ${ }^{2}$ Using two-particle cuts, solutions to the cut conditions ensure that the considered loop momenta remain real and the integrals can be evaluated on those real solutions. However, as is now standard in modern amplitude techniques, considering scattering amplitudes as analytic functions of complex momenta often yields incredible simplifications, allowing us to utilise the full barrage of tools bequeathed to us by complex analysis.

In this spirit, we will revisit the calculation of the classical potential in higher derivative gravity by considering the leading singularity [31,32], the highest codimension singularity of the amplitude, found by fully localizing every loop integral. In doing so, we find that the solutions to the cut conditions are typically complex, and at one loop, this means that the problem of computing loop amplitudes conveniently reduces to the problem of computing residues of some product of (complex) tree amplitudes. It was recently shown that the leading singularity encodes the information required to compute classical gravitational effects $[18,33-35]$, and in this section we will review the techniques required and use them to compute the classical potential in cubic gravity once more, showing that the result is identical to that obtained via unitarity cuts.

\footnotetext{
${ }^{2}$ This process can, however, be almost entirely automated nowadays using one of the many excellent available software packages, for example [30].
} 
Since we require at least one massive propagator in the loop to find classical effects, we consider the triangle diagram in figure 3 . This is due to the fact that we require that the three-point amplitude is present (in this case the all-plus or all-minus helicity vertex). In order to compute the classical piece of this diagram, we will use the on-shell leadingsingularity method presented in [18].

To begin with we will compute the imaginary part of the all-plus contribution to this amplitude, meaning we need to evaluate the integral

$$
I=\sum_{h_{1}, h_{2}} \oint_{\Gamma} \frac{\mathrm{d}^{4} L}{\left(L^{2}-m^{2}\right) k_{1}^{2} k_{2}^{2}} M_{3}\left[P_{1},-L, k_{1}^{-h_{1}}\right] M_{3}\left[L, P_{2}, k_{2}^{-h_{2}}\right] M_{4}\left[-k_{1}^{h_{1}},-k_{2}^{h_{2}}, P_{3}, P_{4}\right],
$$

where $k_{1}=L+P_{1}$ and $k_{2}=L-P_{2}$.

We will parameterise the massive loop momenta by

$$
L=z l+\omega q,
$$

where $z, \omega \in \mathbb{C}$ are parameters to be integrated over, $l=\lambda \tilde{\lambda}$ is massless and $q$ is an arbitrary fixed reference vector.

Cutting the massive propagator and following [18], we can write this as

$$
I=\sum_{h_{1}, h_{2}} \oint_{\Gamma_{L S}} \frac{z \mathrm{~d} z\langle\lambda \mathrm{d} \lambda\rangle[\tilde{\lambda} \mathrm{d} \tilde{\lambda}]}{k_{1}^{2} k_{2}^{2}} M_{3}\left[P_{1},-L, k_{1}^{-h_{1}}\right] M_{3}\left[L, P_{2}, k_{2}^{-h_{2}}\right] M_{4}\left[-k_{1}^{h_{1}},-k_{2}^{h_{2}}, P_{3}, P_{4}\right],
$$

We can also project the external momentum onto the lightcone using massless vectors $p_{1}=\lambda_{1} \tilde{\lambda}_{1}$ and $p_{2}=\lambda_{2} \tilde{\lambda}_{2}$

$$
P_{1}=p_{1}+x p_{2}, \quad P_{2}=p_{2}+x p_{1}, \quad x=\frac{m_{A}^{2}}{2 p_{1} \cdot p_{2}},
$$

where we have used $P_{1}^{2}=P_{2}^{2}=m_{A}^{2}$ to fix $x$. We note that, since we are going to look primarily at the $t$-channel, we can use $x$ to parameterize it as

$$
\frac{(1+x)^{2}}{x}=\frac{t}{m_{A}^{2}}, \quad \frac{(1-x)^{2}}{x}=\frac{t-4 m_{A}^{2}}{m_{A}^{2}} .
$$

If we now also choose two mixed reference vectors $q=\lambda_{1} \tilde{\lambda}_{2}$ and $\bar{q}=\lambda_{2} \tilde{\lambda}_{1}$, then we have four linearly independent vectors which we can use as a basis for our massless loop amplitude, i.e.

$$
l=A p_{1}+B p_{2}+C q+\bar{q} .
$$

Demanding the on-shell condition $l^{2}=0$ gives $C=A B$, and regarding $A, B \in \mathbb{C}$ means we can identify $\mathrm{d} A \mathrm{~d} B \propto\langle\lambda \mathrm{d} \lambda\rangle[\tilde{\lambda} \mathrm{d} \tilde{\lambda}]$. After a change of variables, we find

$$
I=\frac{1}{(2 \pi i)^{3}} \frac{\left(2 p_{1} \cdot p_{2}\right)}{16} \sum_{h_{1}, h_{2}} \oint_{\Gamma_{L S}} \frac{z \mathrm{~d} z \mathrm{~d} A \mathrm{~d} B \quad M_{4}\left[P_{3}, P_{4}, k_{1}^{h_{1}} k_{2}^{h_{2}}\right] M_{3}\left[k_{2}^{-h_{2}}, P_{2}, L\right] M_{3}\left[k_{1}^{-h_{1}}, P_{1},-L\right]}{\left(m_{A}^{2}+z\left(p_{1} \cdot p_{2}\right)(B+x A)\right)\left(-m_{A}^{2}+z\left(p_{1} \cdot p_{2}\right)(A+x B)\right)} .
$$


Using eq. (4.12), we find poles at $A=-B=\frac{2 x}{z(1-x)}$, leaving finally after integration over $A, B$

$$
I=\frac{x}{4 m_{A}^{2}\left(1-x^{2}\right)} \sum_{h_{1}, h_{2}} \frac{1}{2 \pi i} \oint_{\Gamma_{L S}} \frac{\mathrm{d} z}{z} M_{3}\left[P_{1},-L, k_{1}^{-h_{1}}\right] M_{3}\left[L, P_{2}, k_{2}^{-h_{2}}\right] M_{4}\left[-k_{1}^{h_{1}},-k_{2}^{h_{2}}, P_{3}, P_{4}\right] .
$$

With this in hand, we can now parameterize $k_{3}$ and $k_{4}$ using the same parameters. For $k_{3}$, this is

$$
\begin{aligned}
k_{1} & =L+P_{1} \\
& =(1+z A) p_{1}+(z B+x) p_{2}+(z C+\omega) q+z \bar{q} \\
& =r(x) p_{1}-x r(x) p_{2}-\frac{x r^{2}}{z} q+z \bar{q} \\
& =r(x)\left[\lambda_{1}+\frac{z}{r(x)} \lambda_{2}\right]\left[\tilde{\lambda}_{2}-\frac{x}{z} r(x) \tilde{\lambda}_{1}\right],
\end{aligned}
$$

where we have defined $r(x)=\frac{1+x}{1-x}=\left(\frac{t}{t-4 m_{A}^{2}}\right)^{1 / 2}$ and plugged in $\omega=\frac{m_{A}^{2}}{2 z(q \cdot \bar{q})}=-\frac{x}{z}$. Repeating this for $k_{4}$, we find

$$
k_{2}=r(x)\left[\lambda_{2}+\frac{x}{z} r(x) \lambda_{1}\right]\left[\tilde{\lambda}_{2}-\frac{z}{r(x)} \tilde{\lambda}_{1}\right] .
$$

It follows from these parameterizations, that

$$
k_{1} \cdot P_{i}=\frac{1}{z}\left[z^{2} \bar{q} \cdot P_{i}+r(x) z\left(p_{1}-x p_{2}\right) \cdot P_{i}-x r^{2}(x) q \cdot P_{i}\right]
$$

for $i=3,4$. Ultimately, we want to express everything in terms of Mandelstam invariants. Using eq. (4.12), we find that we can write

$$
\begin{aligned}
& \left(p_{1}-x p_{2}\right) \cdot P_{3}=\frac{1}{2}\left(\frac{1+x}{1-x}\right)\left(m_{A}^{2}-m_{B}^{2}+s\right), \\
& \left(p_{1}-x p_{2}\right) \cdot P_{4}=\frac{1}{2}\left(\frac{1+x}{1-x}\right)\left(m_{A}^{2}-m_{B}^{2}+u\right) .
\end{aligned}
$$

As such, eq. (4.19) becomes

$$
\begin{aligned}
& k_{1} \cdot P_{3}=\frac{1}{z}\left[z^{2} \bar{q} \cdot P_{3}+\frac{1}{2} r^{2}(x)\left(m_{A}^{2}-m_{B}^{2}+s\right) z-x r^{2}(x) q \cdot P_{3}\right] \\
& k_{1} \cdot P_{4}=\frac{1}{z}\left[z^{2} \bar{q} \cdot P_{4}+\frac{1}{2} r^{2}(x)\left(m_{A}^{2}-m_{B}^{2}+u\right) z-x r^{2}(x) q \cdot P_{4}\right] .
\end{aligned}
$$

Moreover, as $z$ is our integration variable, we are free to make the following rescaling: $z \longrightarrow$ $2 \frac{1+x}{M^{2}} \sqrt{-x}\left(q \cdot P_{3}\right) z$, where $M$ is defined by $M^{4}:=-4(1-x)^{2}\left(q \cdot P_{3} \bar{q} \cdot P_{3}\right)=\left(m_{A}^{2}-m_{B}^{2}\right)^{2}-s u$ 
(note that $\left(q \cdot P_{3}\right)=-\left(q \cdot P_{4}\right)$, and $\left.\left(q \cdot P_{3} \bar{q} \cdot P_{3}\right)=\left(q \cdot P_{4} \bar{q} \cdot P_{4}\right)\right)$. From this, we find that

$$
\begin{aligned}
& k_{1} \cdot P_{3}=\frac{M^{2} m_{A} r^{2}(x)}{2 \sqrt{-t}} \frac{1}{z}\left[-z^{2}-\left(m_{B}^{2}-m_{A}^{2}-s\right) \frac{\sqrt{-t}}{M^{2} m_{A}} z+1\right]:=\frac{M^{2} m_{A} r^{2}(x)}{2 \sqrt{-t}} \frac{1}{z} F_{s}(z), \\
& k_{1} \cdot P_{4}=\frac{M^{2} m_{A} r^{2}(x)}{2 \sqrt{-t}} \frac{1}{z}\left[z^{2}-\left(m_{B}^{2}-m_{A}^{2}-u\right) \frac{\sqrt{-t}}{M^{2} m_{A}} z-1\right]:=\frac{M^{2} m_{A} r^{2}(x)}{2 \sqrt{-t}} \frac{1}{z} F_{u}(z),
\end{aligned}
$$

where $F_{s}$ and $F_{u}$ are defined as

$$
\begin{aligned}
& F_{s}(z)=-z^{2}-\frac{\sqrt{-t}\left(m_{B}^{2}-m_{A}^{2}-s\right)}{m_{A} \sqrt{-2 m_{A}^{2}\left(m_{B}^{2}+s\right)+m_{A}^{4}-2 s m_{B}^{2}+m_{B}^{4}+s(s+t)}} z+1, \\
& F_{u}(z)=z^{2}-\frac{\sqrt{-t}\left(m_{B}^{2}-m_{A}^{2}-u\right)}{m_{A} \sqrt{-2 m_{A}^{2}\left(m_{B}^{2}+s\right)+m_{A}^{4}-2 s m_{B}^{2}+m_{B}^{4}+s(s+t)}} z-1 .
\end{aligned}
$$

We now need to compute the tree-level amplitudes with which we will build the loop. These are give as follows:

$$
\begin{aligned}
& M_{4}\left[P_{3}, P_{4}, k_{1}^{+}, k_{2}^{+}\right]^{\left(R^{3}\right)}=\frac{3}{64} \kappa^{4} \lambda\left[k_{1} k_{2}\right]^{4} \frac{\beta_{1} t\left(t-2 m_{A}^{2}\right)-16\left(\beta_{1}+2 \beta_{2}\right)\left(k_{1} \cdot P_{3}\right)\left(k_{2} \cdot P_{4}\right)}{\left(P_{3}+P_{4}\right)^{2}}, \\
& M_{4}\left[P_{3}, P_{4}, k_{1}^{+}, k_{2}^{-}\right]^{(G R)}=\frac{\kappa^{2}}{4} \frac{\left[k_{1}\left|P_{3}\right| k_{2}\right\rangle^{2}}{\left(P_{3}+P_{4}\right)^{2}\left[k_{1}\left|P_{3}\right| k_{1}\right\rangle\left[k_{1}\left|P_{4}\right| k_{1}\right\rangle},
\end{aligned}
$$

and,

$$
M_{3}\left[P_{1}, P_{2}, k^{+}\right]^{(G R)}=\frac{\kappa}{2} \frac{\left\langle g\left|P_{1}\right| k\right]^{2}}{\langle g k\rangle^{2}}, \quad M_{3}\left[P_{1}, P_{2}, k^{-}\right]^{(G R)}=\frac{\kappa}{2} \frac{\left[g\left|P_{1}\right| k\right\rangle^{2}}{[g k]^{2}} .
$$

Note that the contributions from cubic gravity (see appendix B for the full derivation) to the four-point amplitudes arise only in the all-positive (and all-negative) helicity case. The reason being is that cubic gravity only affects the three-point vertex function for graviton self-interactions when each graviton has the same helicity (all positive, or all negative).

Focusing on pieces with only one cubic gravity contribution to the loop, and making use of eq. (4.23), one can recast $M_{4}\left[P_{3}, P_{4}, k_{1}^{+}, k_{2}^{+}\right]^{\left(R^{3}\right)}$ into the following form:

$$
\begin{aligned}
M_{4}\left[P_{3}, P_{4}, k_{1}^{+}, k_{2}^{+}\right]^{\left(R^{3}\right)}= & \frac{3}{64} \kappa^{4} \lambda\left[k_{1} k_{2}\right]^{4} \beta_{1}\left(t-2 m_{B}^{2}\right) \\
& +\frac{3}{16 t^{2}} \kappa^{4} \lambda M^{4} m_{A}^{2} r^{4}(x)\left[k_{1} k_{2}\right]^{4} \frac{1}{z^{2}}\left(\beta_{1}+2 \beta_{2}\right) F_{s}(z) F_{u}(z) .
\end{aligned}
$$

To evaluate the leading singularity, we need to plug these tree level amplitudes into eq. (4.16), with $h_{1}=h_{2}=+$, and integrate over the localised integral, i.e. take residues. For this we need to include the product of three-points, given by

$$
\begin{aligned}
M_{3}\left[k_{2}^{-}, P_{2}, L\right]^{(G R)} M_{3}\left[k_{1}^{-}, P_{1},-L\right]^{(G R)} & =\frac{\kappa^{2}}{4} \frac{\left[p_{1}\left|P_{2}\right| k_{2}\right\rangle^{2}}{\left[p_{1} k_{2}\right]^{2}} \frac{\left[p_{2}\left|P_{1}\right| k_{1}\right\rangle^{2}}{\left[p_{2} k_{1}\right]^{2}}=\frac{\kappa^{2}}{4}\left[p_{1} p_{2}\right]^{4} \frac{\left\langle p_{2} k_{2}\right\rangle^{2}\left\langle p_{1} k_{1}\right\rangle^{2}}{\left[p_{1} k_{2}\right]^{2}\left[p_{2} k_{1}\right]^{2}} \\
& =\frac{\kappa^{2}}{4} x^{2} r^{4}(x)\left\langle p_{1} p_{2}\right\rangle^{4}
\end{aligned}
$$


where we have chosen the reference vectors in the three-points to be $p_{1}$ and $p_{2}$, and we have made use of eqs. (4.12), (4.17), and the relations: $\left[p_{1} k_{2}\right]=\left[p_{1} p_{2}\right],\left[p_{1} k_{2}\right]=\frac{x r(x)}{z}\left[p_{1} p_{2}\right]$, $\left\langle p_{1} k_{1}\right\rangle=z\left\langle p_{1} p_{2}\right\rangle$ and $\left\langle p_{2} k_{2}\right\rangle=-\frac{x r^{2(x)}}{z}\left\langle p_{1} p_{2}\right\rangle$.

We will consider the contribution common to both $\beta_{1}$ and $\beta_{2}$ first which, after using definitions of $r(x)$ and $M^{4}$, is given by (using that $\left[k_{1} k_{2}\right]=(1-x)\left[p_{1} p_{2}\right]$ )

$$
\begin{aligned}
I_{++}^{\left(\beta_{1}+2 \beta_{2}\right)} & =\frac{3 \kappa^{6} \lambda m_{A}^{6} M^{4}}{256 t}\left(\frac{t}{t-4 m_{A}^{2}}\right)^{5 / 2} \frac{1}{2 \pi i} \oint_{\Gamma_{L S}} \frac{\mathrm{d} z}{z^{3}} F_{s}(z) F_{u}(z) \\
& =\frac{3 \kappa^{6} \lambda m_{A}^{4}}{256}\left(\frac{t}{t-4 m_{A}^{2}}\right)^{5 / 2}\left[t R\left(s, m_{A}, m_{B}\right)+P\left(s, m_{A}, m_{B}\right)+\frac{Q\left(s, m_{A}, m_{B}\right)}{t}\right] \\
& =I_{--}^{\left(\beta_{1}+2 \beta_{2}\right)}
\end{aligned}
$$

where

$$
\begin{aligned}
& R\left(s, m_{A}, m_{B}\right)=m_{A}^{2}-m_{B}^{2}+s, \\
& P\left(s, m_{A}, m_{B}\right)=2 m_{A}^{2} m_{B}^{2}+\left(s-m_{B}^{2}\right)^{2}-3 m_{A}^{4}, \\
& Q\left(s, m_{A}, m_{B}\right)=2 m_{A}^{2}\left(s-\left(m_{A}+m_{B}\right)^{2}\right)\left(s-\left(m_{A}-m_{B}\right)^{2}\right) .
\end{aligned}
$$

In anticipation of comparison with the unitary cuts calculation, we observe that

$$
c_{3}^{2}(t)=2\left[t R\left(s, m_{A}, m_{B}\right)+P\left(s, m_{A}, m_{B}\right)+\frac{Q\left(s, m_{A}, m_{B}\right)}{t}\right],
$$

and thus

$$
I_{++}^{\left(\beta_{1}+2 \beta_{2}\right)}=-\frac{3 \kappa^{6} \lambda m_{A}^{4}}{512}\left(\frac{t}{t-4 m_{A}^{2}}\right)^{5 / 2} c_{3}^{2}(t) .
$$

The remaining $\beta_{1}$ terms are evaluated analogously, finding

$$
\begin{aligned}
I_{++}^{\beta_{1}} & =\frac{3 \kappa^{6} \lambda m_{A}^{6}}{1024} r^{3}(x) \frac{(1-x)^{2}}{x}\left(t-2 m_{A}^{2}\right) \\
& =\frac{3 \kappa^{6} \lambda m_{A}^{4}}{1024 t}\left(\frac{t}{t-4 m_{A}^{2}}\right)^{5 / 2}\left(t-4 m_{A}^{2}\right)^{2}\left(t-2 m_{B}^{2}\right) \\
& =I_{--}^{\beta_{1}} .
\end{aligned}
$$

Here, we note that $c_{3}^{1}(t)=\frac{1}{2}\left(t-4 m_{A}^{2}\right)^{2}\left(t-2 m_{B}^{2}\right)$, such that $I_{++}^{\beta_{1}}=-\frac{3 \kappa^{6} \lambda m_{A}^{4}}{512 t}\left(\frac{t}{t-4 m_{A}^{2}}\right)^{5 / 2} c_{3}^{1}(t)$.

In general, the full amplitude is related to the imaginary part $I$ by the dispersion relation of a given channel: we integrate the imaginary part of the amplitude along the branch cut in order to reconstruct the entire amplitude [36]. In this case, as was shown in [18], the amplitude has a double discontinuity in the $t$-channel, and we must integrate along both, meaning the full amplitude is given by the dispersion relation

$$
M^{(1)}(s, t)_{++}=\frac{1}{2 \pi i} \int_{0}^{4 m_{A}^{2}} \frac{1}{2 \pi i} \int_{0}^{4 m_{A}^{2}} \frac{\mathrm{d} t^{\prime \prime}}{t^{\prime}-t^{\prime \prime}} \frac{\mathrm{d} t^{\prime}}{t-t^{\prime}} I_{++}\left(s, t^{\prime}\right) .
$$


We need to integrate three integrands: $J, t J$ and $J / t$, where

$$
J(t)=\left(\frac{t}{t-4 m_{A}^{2}+\epsilon}\right)^{5 / 2},
$$

and we have included the $\epsilon$ in order to regulate the divergence. Integrating this (and neglecting terms that vanish in the $\epsilon \longrightarrow 0$ limit), we find

$$
\int_{0}^{4 m_{A}^{2}} \frac{\mathrm{d} t^{\prime}}{t-t^{\prime}} J\left(t^{\prime}\right)=2 \tanh ^{-1}\left(2 \sqrt{\frac{m_{A}^{2}}{\epsilon}} J^{-1 / 5}(t)\right) J(t)-\log \left(\frac{\left(2 m_{A}+\sqrt{\epsilon}\right)^{2}}{\epsilon-4 m_{A}^{2}}\right)+\mathcal{O}\left(\frac{1}{\epsilon^{5 / 2}}\right) .
$$

Keeping only the parts finite in $\epsilon$ and taking $\epsilon \longrightarrow 0$, we find

$$
\int_{0}^{4 m_{A}^{2}} \frac{\mathrm{d} t^{\prime}}{t-t^{\prime}} J\left(t^{\prime}\right)=-i \pi J(t)+i \pi
$$

where we can ignore the addition of $i \pi$ knowing that it comes from the log pieces. ${ }^{3}$ This means that our integral is classically self-similar, and all of the integrations we need to do are therefore trivial, meaning we can write

$$
M^{(1)}(s, t)=\frac{1}{4} \sum_{\beta_{1}, \beta_{2}} \sum_{h_{1}=h_{2}} I_{h_{1} h_{2}}(s, t)+m_{A} \leftrightarrow m_{B}
$$

Evaluating this, we find that the leading singularity is

$$
M_{4}^{(1)}=-\frac{3 \kappa^{6} \lambda m_{A}^{4}}{1024 t}\left(\frac{t}{t-4 m_{A}^{2}}\right)^{5 / 2}\left[\left(\beta_{1}+2 \beta_{2}\right) c_{3}^{2}(t)+\beta_{1} c_{3}^{1}(t)\right]
$$

We see then that the structure of the leading singularity is identical to the classical piece found by directly computing the loop via unitarity cuts. In fact, when evaluating only the classical part of the finite PV integrals, we find that it is given by

$$
\begin{aligned}
C_{0}\left(m_{A}^{2}, m_{A}^{2}, t ; 0, m_{A}, 0\right)_{\text {classical }} & =\frac{\pi^{2}+3 \operatorname{Li}_{2}\left(1+\left(\sqrt{1-\frac{4 m_{A}^{2}}{t}}-1\right) \frac{t}{2 m_{A}^{2}}\right)}{24 \pi^{2} t} \sqrt{\frac{t}{t-4 m_{A}^{2}}} \\
& \simeq \frac{1}{16 t} \sqrt{\frac{t}{t-4 m_{A}^{2}}},
\end{aligned}
$$

where we have kept only the first term in the expansion of $\operatorname{Li}_{2}(1+f[t]) \simeq \frac{\pi^{2}}{6}$.

Plugging this into the unitarity cuts computation and ignoring the quantum corrections show that these match exactly.

Before proceeding, we make a brief comment about extracting the classical contributions to the amplitude (4.37). This can be achieved by appealing to the holomorphic classical limit (HCL) of the amplitude derived via the leading singularity approach (see

\footnotetext{
${ }^{3}$ These only contribute to the quantum piece of the amplitude [37].
} 
ref. [33] for a detailed analysis of the procedure). The HCL corresponds to taking the limit $x \longrightarrow-1$ (i.e. $t / m_{A}^{2} \longrightarrow 0$ ), which in practice means that we should retain only the leading-order-in- $t$ contributions in eq. (4.37). Terms proportional to the same order in $t$ but multiplied by $\log \left(-\frac{t}{m^{2}}\right)$ will therefore be $\propto \hbar$. This is equivalent to the method of restoring factors of $\hbar$ to extricate the classical and quantum components (see [29] for further details on this approach).

\section{$5 \quad$ Potential and black hole solutions}

With the one-loop amplitude in hand, we can now go about deriving the potential. In order to derive the non-relativistic limit, we evaluate the amplitude (4.6), taking the small $t$ limit of the (finite) integrals and summing together with $m_{A} \leftrightarrow m_{B}$. We will focus on the unitarity cuts calculation since this also gives all of the quantum corrections. In the small- $t$ limit, the PV integrals are given by

$$
\begin{aligned}
B_{0}(t) & \simeq \frac{1}{16 \pi^{2}} \log (-t), \\
C_{0}\left(m_{A}^{2}, m_{A}^{2}, t ; 0, m_{A}, 0\right) & \simeq \frac{1}{32 \pi^{2} m_{A}^{2}}\left[\log \left(\frac{m_{A}^{2}}{t}\right)+\frac{\pi^{2} m_{A}}{\sqrt{-t}}\right],
\end{aligned}
$$

Consequently we derive the following amplitude for small $t$ (up to $\mathcal{O}\left(t^{2}\right)$ )

$$
\begin{aligned}
M_{4}^{(1)}= & -\frac{3}{4096} \kappa^{6} \lambda\left(\beta_{1}+2 \beta_{2}\right)\left(m_{A}+m_{B}\right)\left[\left(\left(m_{A}-m_{B}\right)^{2}-s\right)\left(\left(m_{A}+m_{B}\right)^{2}-s\right)(-t)^{3 / 2}\right] \\
& +\frac{3}{1024} \kappa^{6} \lambda \beta_{1}\left[m_{A}^{2} m_{B}^{2}\left(m_{A}+m_{B}\right)(-t)^{3 / 2}\right] \\
& -\frac{3 \hbar}{512 \pi^{2}} \kappa^{6} \lambda\left(\beta_{1}+2 \beta_{2}\right)\left(\left(m_{A}^{2}-s\right)^{2}+\left(m_{B}^{2}-s\right)^{2}-s^{2}\right) t^{2} \log (-t) \\
& +\frac{3 \hbar}{512 \pi^{2}} \kappa^{6} \lambda \beta_{1} m_{A}^{2} m_{B}^{2} t^{2} \log (-t)
\end{aligned}
$$

We can take the fully non-relativistic limit of this via ${ }^{4}$

$$
\begin{aligned}
& t \longrightarrow-\mathbf{q}^{2}, \\
& s \longrightarrow\left(m_{A}+m_{B}\right)^{2}\left(1+\frac{\mathbf{p}^{2}+\frac{1}{4} \mathbf{q}^{2}}{m_{A} m_{B}}\right),
\end{aligned}
$$

which leaves us with a momentum space potential

$$
V(\mathbf{q}, \mathbf{p})=V_{\mathrm{cl}}(\mathbf{q}, \mathbf{p})+\hbar V_{\mathrm{qu}}(\mathbf{q}, \mathbf{p})
$$

\footnotetext{
${ }^{4}$ We thank the authors of [38] for very useful discussions on this point.
} 
where $V_{\mathrm{cl}}(\mathbf{q})$ and $V_{\mathrm{qu}}(\mathbf{q})$ are the classical and quantum contributions, respectively given by

$$
\begin{aligned}
V_{\mathrm{cl}}(\mathbf{q}, \mathbf{p})= & -\frac{3}{4096} \kappa^{6} \lambda\left(\beta_{1}+2 \beta_{2}\right)\left[\frac{\left(m_{A}+m_{B}\right)^{3}}{m_{A} m_{B}} \mathbf{p}^{2}|\mathbf{q}|^{3}\right] \\
& +\frac{3}{8192} \kappa^{6} \lambda \beta_{1} \frac{\left(m_{A}+m_{B}\right)}{m_{A} m_{B}}\left[2 m_{A}^{2} m_{B}^{2}|\mathbf{q}|^{3}-\left(m_{A}^{2}+m_{B}^{2}\right) \mathbf{p}^{2}|\mathbf{q}|^{3}\right]+\mathcal{O}\left(|\mathbf{q}|^{5}\right) \\
V_{\mathrm{qu}}(\mathbf{q}, \mathbf{p})= & -\frac{3}{2048 \pi^{2}} \kappa^{6} \lambda \frac{\left(\beta_{1}+2 \beta_{2}\right)}{m_{A} m_{B}}\left[2 m_{A}^{2} m_{B}^{2}+\left(3 m_{A}^{2}+8 m_{A} m_{B}+3 m_{B}^{2}\right) \mathbf{p}^{2}\right] \mathbf{q}^{4} \log \left(\mathbf{q}^{2}\right) \\
& +\frac{3}{4096 \pi^{2}} \kappa^{6} \lambda \beta_{1}\left[2 m_{A} m_{B}-\frac{\left(m_{A}^{2}+m_{B}^{2}\right)}{m_{A} m_{B}} \mathbf{p}^{2}\right] \mathbf{q}^{4} \log \left(\mathbf{q}^{2}\right)+\mathcal{O}\left(|\mathbf{q}|^{6}\right)
\end{aligned}
$$

Taking the Fourier transform as in appendix B, we find

$$
V(\mathbf{r}, \mathbf{p})=V_{\mathrm{cl}}(\mathbf{r}, \mathbf{p})+\hbar V_{\mathrm{qu}}(\mathbf{r}, \mathbf{p}),
$$

where

$$
\begin{aligned}
V_{\mathrm{cl}}(\mathbf{r}, \mathbf{p})= & \frac{9}{1024 \pi^{2}} \kappa^{6} \lambda\left(\beta_{1}+2 \beta_{2}\right)\left[\frac{\left(m_{A}+m_{B}\right)^{3}}{m_{A} m_{B}} \frac{\mathbf{p}^{2}}{r^{6}}\right] \\
& -\frac{9}{2048 \pi^{2}} \kappa^{6} \lambda \beta_{1}\left(m_{A}+m_{B}\right)\left[2 \frac{m_{A} m_{B}}{r^{6}}-\frac{\left(m_{A}^{2}+m_{B}^{2}\right)}{m_{A} m_{B}} \frac{\mathbf{p}^{2}}{r^{6}}\right]+\mathcal{O}\left(r^{-8}\right), \\
V_{\mathrm{qu}}(\mathbf{r}, \mathbf{p})= & -\frac{45}{512 \pi^{3}} \kappa^{6} \lambda\left(\beta_{1}+2 \beta_{2}\right)\left[\frac{2 m_{A} m_{B}}{r^{7}}+\frac{\left(3 m_{A}^{2}+8 m_{A} m_{B}+3 m_{B}^{2}\right)}{m_{A} m_{B}} \frac{\mathbf{p}^{2}}{r^{7}}\right] \\
& +\frac{45}{1024 \pi^{3}} \kappa^{6} \lambda \beta_{1}\left[2 \frac{m_{A} m_{B}}{r^{7}}-\frac{\left(m_{A}^{2}+m_{B}^{2}\right)}{m_{A} m_{B}} \frac{\mathbf{p}^{2}}{r^{7}}\right]+\mathcal{O}\left(r^{-9}\right) .
\end{aligned}
$$

Equipped with the expressions for the classical and quantum corrections to the potential, we can make contact with some specific theories my making particular choices for the couplings. Firstly, we derive the first order corrections to the potential arising from ECG, by choosing $\beta_{1}=12, \beta_{2}=1$ and restoring $G$ via $\kappa=\sqrt{32 \pi G}$. Doing so gives the potential

$$
V_{\mathrm{ECG}}(\mathbf{r})=72 \tilde{\lambda} G^{4} m_{A} m_{B}\left(\frac{3\left(m_{A}+m_{B}\right)}{r^{6}}-\hbar \frac{100}{\pi r^{7}}\right) .
$$

where we have rescaled the coupling by

$$
\lambda \longrightarrow-\frac{G \tilde{\lambda}}{16 \pi},
$$

in order to ensure that our $\lambda$ matches the one in ref. [16].

We can also compute the corrections to the potential that arise from the $\alpha^{\prime 2}$ part of low-energy effective action in string theory, recently discussed in [38], which enter as

$$
S=-\frac{2 \alpha^{\prime 2}}{\kappa^{2}} \int d^{4} x \sqrt{-g}\left(\frac{1}{48} I_{1}+\frac{1}{24} G_{3}\right)
$$


where $I_{1}$ is the $\beta_{2}$ term in our case. To compute the $I_{1}$ correction, we take $\lambda=-\frac{2 \alpha^{\prime 2}}{\kappa^{2}}$ and then $\beta_{1}=0, \beta_{2}=\frac{1}{48}$ which gives

$$
V_{I_{1}}(\mathbf{r})=3\left(\alpha^{\prime} G\right)^{2}\left(\frac{\left(m_{A}+m_{B}\right)^{3}}{4 m_{A} m_{B}} \frac{\mathbf{p}^{2}}{r^{6}}-\hbar \frac{5 m_{A} m_{B}}{\pi r^{7}}\right)
$$

Then to compute the $G_{3}$ contribution we take $\beta_{1}=-2 \beta_{2}=-\frac{1}{24}$ to find

$$
V_{G_{3}}(\mathbf{r})=\frac{3\left(\alpha^{\prime} G\right)^{2}}{4} m_{A} m_{B}\left(\frac{\left(m_{A}+m_{B}\right)}{r^{6}}-\hbar \frac{10}{\pi r^{7}}\right) .
$$

Furthermore, given the potential we can, as discussed in section 3 above, derive a static, spherically symmetric black hole solution. Knowing the form of the Schwarzschild solution, and noting the argument earlier that any solution derived from a cubic theory must be a correction to this, we find a black hole solution of the form

$$
f(\mathbf{r})=1-\frac{2 G m_{A}}{r}-36 \beta_{1} \frac{G^{4} \tilde{\lambda} m_{A}^{2}}{r^{6}}+360 \hbar\left(3 \beta_{1}+4 \beta_{2}\right) \frac{G^{4} \tilde{\lambda} m_{A}}{\pi r^{7}} .
$$

Choosing the specific coefficients in ECG, we find a solution of the form

$$
f(\mathbf{r})=1-\frac{2 G m_{A}}{r}-432 \frac{G^{4} \tilde{\lambda} m_{A}^{2}}{r^{6}}+14400 \hbar \frac{G^{4} \tilde{\lambda} m_{A}}{\pi r^{7}} .
$$

The classical part of this metric matches those derived from Einsteinian cubic gravity $[14,39]$.

The solutions found in those papers were not easy to come by, being the (perturbative) solution to a particularly complicated differential equation with apparently no analytic solution. Here, we have come to the same solution by considering gravity as a quantum field theory and using the tools of modern scattering amplitudes, deriving the quantum corrections to the metric as an added bonus. Furthermore, we showed how the same classical black hole solution could be obtained by computing residues of the leading singularity.

\section{Discussion}

In this paper, we have studied the leading order dynamics of a general cubic theory of gravity coupled to a spin-zero matter field, within the framework of the modern scattering amplitude techniques.

With a view to determining the effects of cubic gravity on the purely classical graviton mediated interaction between two scalars, we observed that this can only occur at one-loop order and above, at least when considering minimal coupling. It is known that loops can provide classical contributions [27, 29], and moreover, that any classical contributions to the gravitational potential manifest in diagrams containing massive propagators. Given this, we computed the double cut of the appropriate one-loop diagram, in which we cut two internal graviton lines and retaining only the leading order contributions to the result.

We then repeated this calculation by considering an alternative approach: computing the leading singularity of the one-loop triangle diagram with one massive propagator. Not 
only was this a much more straightforward calculation, importantly, we recovered the result we found using the standard unitarity cuts method. This provides us with confidence that the final result that we obtained is consistent. Indeed, whilst this draft was in preparation, Brandhuber and Travaglini published work in which they consider the (dynamically) nontrivial cubic corrections to the gravitational action arising from string theory [38]. Upon comparison of the two sets of results, we find that the corresponding cubic modification of the gravitational potential agrees, up to an additional non-dispersive contribution, with theirs. The most interesting difference between our results is this additional contribution, since its structure is such that, in an appropriate probe-limit, we were able to derive a black hole solution which exactly corresponds to the leading order Einsteinian cubic gravity contribution, matching the result found in ref. [14]. It is interesting to note that this black hole solution survives the limit where the cubic gravity theory under consideration is that of pure cubic Gauss-Bonnet, $G_{3}$, and that the black hole solution arises from the nonminimal coupling between the spin-zero matter field and the Gauss-Bonnet combination. This is not wholly unexpected, since although possessing trivial dynamics in isolation, it has been shown that the Gauss-Bonnet combination has non-trivial effects on four-point amplitudes when coupled the matter sector [19, 20].

\section{Acknowledgments}

We would like to thank Daniel Burger, Raúl Carballo-Rubio, Alfredo Guevara, Jeff Murugan, and Amanda Weltman for useful discussions during the preparation of this work. WTE would like to thank Amanda in particular for hosting him at UCT for the majority of this project. In addition, WTE acknowledges support from the South African Research Chairs Initiative for supporting this visit. NM would like to thank Imperial College London and Nottingham University for hosting him while some of this work was completed. NM is supported by the South African Research Chairs Initiative of the Department of Science and Technology and the National Research Foundation of South Africa. Any opinion, finding and conclusion or recommendation expressed in this material is that of the authors and the NRF does not accept any liability in this regard.

\section{A Tree-level 4-point amplitude}

To derive the current needed to compute amplitudes involving cubic invariants, we expand the following around flat space (using xAct [40])

$$
\mathcal{P}=\sqrt{-g}\left[\beta_{1} R_{a b}^{c d} R_{c d}^{e f} R_{e f}^{a b}+\beta_{2} R_{a b}^{c d} R_{c d}^{e f} R_{e f}^{a b}+\beta_{3} R_{a b c d} R^{a c} R^{b d}+\beta_{4} R_{a}^{b} R_{b}^{c} R_{c}^{a}\right] .
$$

Expanding to cubic order and including a de Donder gauge-fixing term, we find that only the terms with coefficient $\beta_{1}$ and $\beta_{2}$ survive the requirements that the polarization tensors be transverse (i.e. that $k_{\mu} \epsilon^{\mu}(k)=0$ ) and the on-shell condition $k^{2}=0$. Since terms of cubic order only contribute same-helicity 3-points, we put two of the legs of the 3-point on shell and fix their helicity to both be identical. Factoring out the third leg, we derive 
the following currents

$$
\begin{aligned}
J_{--,--}^{\mu \nu}=\kappa^{3} \lambda \frac{3}{16}\langle 12\rangle^{4} & {\left[\beta_{1}\left(k_{1}^{\mu} k_{1}^{\nu}+k_{2}^{\mu} k_{2}^{\nu}-k_{1}^{\mu} k_{2}^{\nu}-k_{2}^{\mu} k_{1}^{\nu}\right)\right.} \\
& \left.-\beta_{2}\left(\left\langle 1\left|\gamma^{\mu}\right| 2\right]\left\langle 2\left|\gamma^{\nu}\right| 1\right]+\left\langle 1\left|\gamma^{\nu}\right| 2\right]\left\langle 2\left|\gamma^{\mu}\right| 1\right]\right)\right], \\
J_{++,++}^{\mu \nu}=\kappa^{3} \lambda \frac{3}{16}[12]^{4}[ & \beta_{1}\left(k_{1}^{\mu} k_{1}^{\nu}+k_{2}^{\mu} k_{2}^{\nu}-k_{1}^{\mu} k_{2}^{\nu}-k_{2}^{\mu} k_{1}^{\nu}\right) \\
& \left.-\beta_{2}\left(\left\langle 2\left|\gamma^{\mu}\right| 1\right]\left\langle 1\left|\gamma^{\nu}\right| 2\right]+\left\langle 2\left|\gamma^{\nu}\right| 1\right]\left\langle 1\left|\gamma^{\mu}\right| 2\right]\right)\right] .
\end{aligned}
$$

The scalar-scalar-graviton vertex, and the graviton propagator (in the de Donder gauge) are further given by, respectively

$$
J_{00}^{\mu \nu}=-\frac{\kappa}{2}\left[P_{3}^{\mu} P_{4}^{\nu}+P_{3}^{\nu} P_{4}^{\mu}-\eta^{\mu \nu}\left(P_{3} \cdot P_{4}+m^{2}\right)\right],
$$

and

$$
P_{\mu_{1} \nu_{1} ; \mu_{2} \nu_{2}}=-\frac{1}{2 k^{2}}\left[\eta_{\mu_{1} \mu_{2}} \eta_{\nu_{1} \nu_{2}}+\eta_{\mu_{1} \nu_{2}} \eta_{\nu_{1} \mu_{2}}-\eta_{\mu_{1} \nu_{1}} \eta_{\mu_{2} \nu_{2}}\right]
$$

Contracting these with $J_{--,--}^{\mu \nu}\left(\right.$ and $\left.J_{00}^{\mu \nu}\right)$, we derive the 4-points

$$
\begin{aligned}
& M_{4}\left[k_{1}^{-}, k_{2}^{-}, P_{3}, P_{4}\right]=\frac{3}{16} \kappa^{4} \lambda\langle 12\rangle^{4} \frac{\beta_{1}\left(\left(k_{2} \cdot P_{3}-k_{2} \cdot P_{4}\right)^{2}-m^{2} k_{1} \cdot k_{2}\right)-8 \beta_{2}\left(k_{1} \cdot P_{3}\right)\left(k_{2} \cdot P_{3}\right)}{\left(P_{3}+P_{4}\right)^{2}} \\
& M_{4}\left[k_{1}^{+}, k_{2}^{+}, P_{3}, P_{4}\right]=\frac{3}{16} \kappa^{4} \lambda[12]^{4} \frac{\beta_{1}\left(\left(k_{2} \cdot P_{3}-k_{2} \cdot P_{4}\right)^{2}-m^{2} k_{1} \cdot k_{2}\right)-8 \beta_{2}\left(k_{1} \cdot P_{3}\right)\left(k_{2} \cdot P_{3}\right)}{\left(P_{3}+P_{4}\right)^{2}}
\end{aligned}
$$

where, in the case of ECG, $\beta_{1}=12$ and $\beta_{2}=1$. Aside from this, if we take $\beta_{1}=-2 \beta_{2}$ then the 4-point amplitudes above remain non-trivial, induced by a cubic Gauss-Bonnet $G_{3}$-interaction. In this case, they reduce to

$$
\begin{aligned}
& \frac{1}{\lambda} M_{4}\left[k_{1}^{-}, k_{2}^{-}, P_{3}, P_{4}\right]^{\left(G_{3}\right)}=\frac{3 !}{4}\left(\frac{\kappa}{2}\right)^{4}\langle 12\rangle^{4}\left(t+2 m^{2}\right), \\
& \frac{1}{\lambda} M_{4}\left[k_{1}^{+}, k_{2}^{+}, P_{3}, P_{4}\right]^{\left(G_{3}\right)}=\frac{3 !}{4}\left(\frac{\kappa}{2}\right)^{4}[12]^{4}\left(t+2 m^{2}\right),
\end{aligned}
$$

in agreement with the result found in [38].

\section{B Fourier transforms}

We need to compute the Fourier transform of $|\mathbf{q}|^{n}$, where $n$ is positive

$$
F[\mathbf{r}, n]=\int \frac{\mathrm{d}^{3} \mathbf{q}}{(2 \pi)^{3}} e^{i \mathbf{q} \cdot \mathbf{r}}|\mathbf{q}|^{n}
$$


Formally, this diverges and requires regularization. To do so, we shift $\mathbf{q}$ by a regulator $\epsilon$, i.e.

$$
F[\mathbf{r}, n]=\int \frac{\mathrm{d}^{3} \mathbf{q}}{(2 \pi)^{3}} e^{i \mathbf{q} \cdot \mathbf{r}-\epsilon|\mathbf{q}| r}|\mathbf{q}|^{n},
$$

where $\epsilon \ll 1$ and we discard higher orders. Switching to spherical-polar coordinates, this becomes

$$
F[\mathbf{r}, n]=\int_{0}^{\pi} \mathrm{d} \theta \int \frac{\mathrm{d}|\mathbf{q}|}{(2 \pi)^{3}} e^{i|\mathbf{q}| r \cos \theta-\epsilon|\mathbf{q}| r}|\mathbf{q}|^{n+2} \sin \theta,
$$

Integrating this with $\epsilon \longrightarrow 0$, we can define an identity valid for odd integers $n$, satisfying $n \geq-1$

$$
\int \frac{\mathrm{d}^{3} \mathbf{q}}{(2 \pi)^{3}} e^{i \mathbf{q} \cdot \mathbf{r}}|\mathbf{q}|^{n}=\frac{(n+1) !}{2 \pi^{2} r^{3+n}} \sin \left(\frac{3 \pi n}{2}\right) .
$$

Including a log piece and repeating the same procedure yields a similar but unfortunately more unwieldy identity, and so we simply note only the following identities

$$
\begin{aligned}
& \int \frac{\mathrm{d}^{3} \mathbf{q}}{(2 \pi)^{3}} e^{i \mathbf{q} \cdot \mathbf{r}}|\mathbf{q}|^{4} \log \left(\mathbf{q}^{2}\right)=-\frac{60}{\pi r^{7}} \\
& \int \frac{\mathrm{d}^{3} \mathbf{q}}{(2 \pi)^{3}} e^{i \mathbf{q} \cdot \mathbf{r}}|\mathbf{q}|^{6} \log \left(\mathbf{q}^{2}\right)=\frac{2520}{\pi r^{9}}
\end{aligned}
$$

Open Access. This article is distributed under the terms of the Creative Commons Attribution License (CC-BY 4.0), which permits any use, distribution and reproduction in any medium, provided the original author(s) and source are credited.

\section{References}

[1] J. Bedford, A. Brandhuber, B.J. Spence and G. Travaglini, A Recursion relation for gravity amplitudes, Nucl. Phys. B 721 (2005) 98 [hep-th/0502146] [INSPIRE].

[2] D. Nguyen, M. Spradlin, A. Volovich and C. Wen, The Tree Formula for MHV Graviton Amplitudes, JHEP 07 (2010) 045 [arXiv:0907.2276] [INSPIRE].

[3] P. Benincasa, C. Boucher-Veronneau and F. Cachazo, Taming Tree Amplitudes In General Relativity, JHEP 11 (2007) 057 [hep-th/0702032] [INSPIRE].

[4] N.E.J. Bjerrum-Bohr, P.H. Damgaard, G. Festuccia, L. Planté and P. Vanhove, General Relativity from Scattering Amplitudes, Phys. Rev. Lett. 121 (2018) 171601 [arXiv: 1806. 04920] [INSPIRE].

[5] H. Johansson and J. Nohle, Conformal Gravity from Gauge Theory, arXiv:1707.02965 [INSPIRE].

[6] S. He and Y. Zhang, New Formulas for Amplitudes from Higher-Dimensional Operators, JHEP 02 (2017) 019 [arXiv: 1608.08448] [INSPIRE].

[7] D.C. Dunbar, J.H. Godwin, G.R. Jehu and W.B. Perkins, Diagrammar in an Extended Theory of Gravity, Phys. Lett. B 771 (2017) 230 [arXiv:1702.08273] [INSPIRE].

[8] D.C. Dunbar, J.H. Godwin, G.R. Jehu and W.B. Perkins, Loop Amplitudes in an Extended Gravity Theory, Phys. Lett. B 780 (2018) 41 [arXiv:1711.05526] [INSPIRE]. 
[9] R. Carballo-Rubio, F. Di Filippo and N. Moynihan, Taming higher-derivative interactions and bootstrapping gravity with soft theorems, JCAP 10 (2019) 030 [arXiv:1811.08192] [INSPIRE].

[10] C. Cheung, I.Z. Rothstein and M.P. Solon, From Scattering Amplitudes to Classical Potentials in the Post-Minkowskian Expansion, Phys. Rev. Lett. 121 (2018) 251101 [arXiv: 1808.02489] [INSPIRE].

[11] Z. Bern, C. Cheung, R. Roiban, C.-H. Shen, M.P. Solon and M. Zeng, Scattering Amplitudes and the Conservative Hamiltonian for Binary Systems at Third Post-Minkowskian Order, Phys. Rev. Lett. 122 (2019) 201603 [arXiv:1901. 04424] [INSPIRE].

[12] T. Damour, Gravitational scattering, post-Minkowskian approximation and Effective One-Body theory, Phys. Rev. D 94 (2016) 104015 [arXiv:1609.00354] [InSPIRE].

[13] T. Damour, High-energy gravitational scattering and the general relativistic two-body problem, Phys. Rev. D 97 (2018) 044038 [arXiv:1710.10599] [InSPIRE].

[14] P. Bueno and P.A. Cano, Four-dimensional black holes in Einsteinian cubic gravity, Phys. Rev. D 94 (2016) 124051 [arXiv: 1610.08019] [INSPIRE].

[15] A.A. Tseytlin, Vector Field Effective Action in the Open Superstring Theory, Nucl. Phys. B 276 (1986) 391 [Erratum ibid. B 291 (1987) 876] [INSPIRE].

[16] P. Bueno and P.A. Cano, Einsteinian cubic gravity, Phys. Rev. D 94 (2016) 104005 [arXiv: 1607.06463] [INSPIRE].

[17] I. Güllü, T.C. Sisman and B. Tekin, Born-Infeld Gravity with a Massless Graviton in Four Dimensions, Phys. Rev. D 91 (2015) 044007 [arXiv:1410.8033] [InSPIRE].

[18] F. Cachazo and A. Guevara, Leading Singularities and Classical Gravitational Scattering, arXiv: 1705.10262 [INSPIRE].

[19] R.R. Metsaev and A.A. Tseytlin, Curvature Cubed Terms in String Theory Effective Actions, Phys. Lett. B 185 (1987) 52 [INSPIRE].

[20] J. Broedel and L.J. Dixon, Color-kinematics duality and double-copy construction for amplitudes from higher-dimension operators, JHEP 10 (2012) 091 [arXiv:1208.0876] [INSPIRE].

[21] K.A. Kazakov, On the notion of potential in quantum gravity, Phys. Rev. D 63 (2001) 044004 [hep-th/0009220] [INSPIRE].

[22] Y. Iwasaki, Fourth-order gravitational potential based on quantum field theory, Lett. Nuovo Cim. 1 (1971) 783 [INSPIRE].

[23] Y. Iwasaki, Quantum Theory of Gravitation vs. Classical Theory: Fourth-Order Potential, Prog. Theor. Phys. 46 (1971) 1587 [InSPIRE].

[24] M.J. Duff, Quantum corrections to the Schwarzschild solution, Phys. Rev. D 9 (1974) 1837 [INSPIRE].

[25] D. Neill and I.Z. Rothstein, Classical Space-Times from the S Matrix, Nucl. Phys. B 877 (2013) 177 [arXiv:1304.7263] [inSPIRE].

[26] G. Modanese, Potential energy in quantum gravity, Nucl. Phys. B 434 (1995) 697 [hep-th/9408103] [INSPIRE].

[27] B.R. Holstein and J.F. Donoghue, Classical physics and quantum loops, Phys. Rev. Lett. 93 (2004) 201602 [hep-th/0405239] [INSPIRE]. 
[28] J.F. Donoghue, General relativity as an effective field theory: The leading quantum corrections, Phys. Rev. D 50 (1994) 3874 [gr-qc/9405057] [INSPIRE].

[29] D.A. Kosower, B. Maybee and D. O'Connell, Amplitudes, Observables and Classical Scattering, JHEP 02 (2019) 137 [arXiv:1811.10950] [INSPIRE].

[30] H.H. Patel, Package-X: A Mathematica package for the analytic calculation of one-loop integrals, Comput. Phys. Commun. 197 (2015) 276 [arXiv:1503.01469] [INSPIRE].

[31] N. Arkani-Hamed, F. Cachazo and J. Kaplan, What is the Simplest Quantum Field Theory?, JHEP 09 (2010) 016 [arXiv: 0808.1446] [INSPIRE].

[32] R. Britto, F. Cachazo and B. Feng, Generalized unitarity and one-loop amplitudes in $N=4$ super-Yang-Mills, Nucl. Phys. B 725 (2005) 275 [hep-th/0412103] [INSPIRE].

[33] A. Guevara, Holomorphic Classical Limit for Spin Effects in Gravitational and Electromagnetic Scattering, JHEP 04 (2019) 033 [arXiv:1706.02314] [INSPIRE].

[34] A. Guevara, A. Ochirov and J. Vines, Scattering of Spinning Black Holes from Exponentiated Soft Factors, JHEP 09 (2019) 056 [arXiv: 1812.06895] [INSPIRE].

[35] Y.F. Bautista and A. Guevara, From Scattering Amplitudes to Classical Physics: Universality, Double Copy and Soft Theorems, arXiv:1903.12419 [INSPIRE].

[36] J.F. Donoghue, Dispersion relations and effective field theory, in proceedings of the Advanced School on Effective Theories, Almunecar, Spain, 25 June-1 July 1995, hep-ph/9607351 [INSPIRE].

[37] B.R. Holstein and A. Ross, Spin Effects in Long Range Gravitational Scattering, arXiv:0802.0716 [INSPIRE].

[38] A. Brandhuber and G. Travaglini, On higher-derivative effects on the gravitational potential and particle bending, arXiv:1905.05657 [INSPIRE].

[39] R.A. Hennigar and R.B. Mann, Black holes in Einsteinian cubic gravity, Phys. Rev. D 95 (2017) 064055 [arXiv:1610.06675] [INSPIRE].

[40] J.M. Martin-Garcia, R. Portugal and L.R.U. Manssur, The Invar Tensor Package, Comput. Phys. Commun. 177 (2007) 640 [arXiv:0704.1756] [INSPIRE]. 